\title{
Investigations into the free-volume changes within starch/plasticizer/ nanoclay systems using Positron Annihilation Lifetime Spectroscopy
}

\author{
Huihua Liu ${ }^{\text {a, * }}$, Deeptangshu Chaudhary ${ }^{\text {b }}$, Colin Campbell ${ }^{\text {c }}$, Jason Roberts ${ }^{\text {, }}$, \\ Stephen Buckman ${ }^{\mathrm{C}}$, James Sullivan ${ }^{\mathrm{c}}$ \\ a School of Health Sciences, Federation University Australia, Ballarat, Vic, Australia \\ ${ }^{\mathrm{b}}$ ERS Environmental Risk Solutions PTY LTD, Perth, WA, Australia \\ c ARC Centre of Excellence for Antimatter-Matter Studies (CAMS), Research School of Physics and Engineering, Australian National University, \\ Canberra, Australia
}

\section{H I G H L I G H T S}

- PALS is applied to explore the synergistic interaction from a molecular level.

- Quantitative data on free volume radii and distribution were extracted via PALS.

- Relationship of Free volume changes and bulk properties evidenced.

- The total free-volume is a reflection of level of interactions existing.

\section{A R T I C L E I N F O}

\section{Article history:}

Received 15 October 2013

Received in revised form 29 April 2014

Accepted 28 July 2014

Available online 15 August 2014

\section{Keywords:}

Nanostructures

Small-angle scattering

Positron annihilation spectroscopy

Domain structure

\begin{abstract}
A B S T R A C T
The free-volume of a matrix is a fundamental parameter that relates to its molecular and bulk characteristics, such as crystalline change and glass transition behavior. In starch-based bionanocomposite, we investigated the effect of the addition of montmorillonite nanoclay (MMT) and food plasticizers (glycerol and sorbitol) on changes of molecular pore size (including pore volume and pore distribution) using the Positron Annihilation Lifetime Spectroscopy (PALS) method. The results demonstrated counter-intuitive impact of MMT on the total free-volume where the total free-volume increased within the polymeric matrix. When compared to the pure matrix free-volume, the addition of MMT also resulted in the appearance of a broader distribution of the void sizes. The plasticizers, on the other hand, apparently occupied the void spaces, and therefore decreased the free-volume of the matrix. Further, together with the small angle X-ray scattering (SAXS) analysis, we concluded that this is a result of interplay between the plasticizer-plasticizer interactions and the polymer-plasticizer interactions. For example, in the starch/glycerol/MMT system, the pore radii slightly decrease upon the increasing of glycerol amount $(0 G 210=0.27 \mathrm{~nm}$ and $0 G 220=0.26 \mathrm{~nm})$, but the relative weight did increase with the increasing glycerol concentration. However, increasing the sorbitol amount increased the pore size from $0.23 \mathrm{~nm}(\mathrm{OS} 210)$ to $0.28 \mathrm{~nm}(\mathrm{OS} 220)$. Furthermore, the addition MMT in the OS010 system, promote the emergence of a new dateable pore $\operatorname{radius}(0.90 \mathrm{~nm})$, and the total weight significantly increased from 13.70 (OS010) to $19.5 \%$ (OS210). We suggest that the pore variation (size and distribution) due to the MMT and plasticizers are reflected in the polymer glass transition and crystallinity because ultimately, the, total free-volume is a reflection of level of interactions existing within the bulk of these nanocomposites.
\end{abstract}

๑) 2014 Elsevier B.V. All rights reserved.

\footnotetext{
* Corresponding author. Tel.: +61 353279942.

E-mail addresses: h.liu@federation.edu.au, amyliuscut@gmail.com (H. Liu), deepc@ers.com.au (D. Chaudhary), colin.campbell@anu.edu.au (C. Campbell), jxr107@physics.anu.edu.au（J. Roberts), stephen.buckman@anu.edu.au (S. Buckman), james.sullivan@anu.edu.au (J. Sullivan).
}

\section{Introduction}

Biodegradable materials, such as starch based bionanocomposites have shown some commercial success in the area of flexible packaging, but are still limited in their potential due to their moisture sensitivity and matrix crystallinity. As a result, plasticizer molecules and nanoparticles are added to the starch 
polymer so as to improve the polymer's moisture sensitivity. Because these additives have hydrophilic interactions with the starch matrix, binary and ternary level interactions exist in the starch bionanocomposite system, which can directly influence the polymeric chains' movement and matrix morphology; and thus affect the bulk crystallinity, glass transition $\left(T_{\mathrm{g}}\right)$ and moisture retention behavior [1,2]. From several previous publications, it's believed that the structure and organization of the polymeric network, mainly in terms of the crystalline domain and matrix freevolume, are influenced by the concentration and the interactions strength of the plasticizers and the nanoclays(MMT) [1,3,4]. In fact, it was reported earlier that the presence of MMT altered the morphologies and the crystalline size domains within the polymeric network. The same study also reported that the presence of plasticizer molecule reinforced the long range crystalline order and greater heterogeneity within the polymer matrix [5].

Since the organization of the polymeric chains and the crystalline domain would strongly influence the free-volume of a polymeric system [6,7], it is therefore of great significance to investigate (1) how would the free-volume of this bionanocomposite system alter and further, (2) how would the $T_{\mathrm{g}}$ fluctuate as the free-volume changes. The interaction among starch/plasticizer/MMT enables polymer segregation or coalescence, and the impact of this phenomenon on the overall free-volume of the matrix can be captured quantitatively using the Positron Annihilation Lifetime Spectroscopy (PALS) technique. The PALS is an important technique for measuring polymer free-volume [8]. In polymer studies, two important parameters are measured by PALS; the lifetime of ortho-positronium (oPs), $\tau$, and the intensity of the oPs signal, I. These parameters are related to the mean free-volume radius - or "cavity". These two parameters in polymer science could be treated as the unoccupied space between and along polymer chains and/for the relative concentration of such sites, respectively. A systematical study of the free-volume variation in these polymeric systems will provide significant insights into exploring any complex interactions within the nanocomposites. In a PALS measurement, by observing the lifetime of the positrons in the material, we can get information about the size and distribution of the open volume. This quantitative information can then be related to micro- and macro- properties of the material, such as conductivity, gas diffusion, and crystalline domains.

This study aims to understand the molecular-scale interaction in the starch-based nanocomposites by using PALS measurements via comparing the polymeric pore size changes and distributions across different samples with specific formulation. To our knowledge, this is the first such quantification of molecular pore volume within starch based bionanocomposites, which provides an understanding of how the free-volume is modified by plasticizer and MMT. The recent work of Roussenova et al. investigated a glycerol-plasticized system using maltodextrin as a reference for the changes in freevolume [9]. However, since maltodextrin is only an oligomer of the glucose unit, it's useful to have a full-scale investigation of the original matrix with the industrially relevant plasticizers. Moreover, we have used Small Angle X-ray Scattering (SAXS) and Differential Scanning Calorimetry (DSC) techniques to complement and corroborate the findings from the PALS analysis. Further to understand the influence of type of additive, we have discussed the results of a binary system (starch/MMT and starch/plasticizer) and compared it with ternary(starch/plasticizer/MMT) systems.

\section{Materials and method}

\subsection{Materials and extrusion}

High Amylose starch (80\% amylose) was purchased from National Starch Company (New Jersey, USA); glycerol and sorbitol was obtained from Food Dept Melbourne Company (Melbourne, Australia); montmorillonite nanoclay (MMT) (99.5\% purity) was supplied by NichePlas Ltd. (Sydney, Australia). Ten samples were prepared at different ratios of $\mathrm{MMT} /$ plasticizers content via extrusion (detailed processing parameters, please refer to our previous report Ref [10]). Nomenclatures used, such as OS210, are listed in Table 1, where 'OS2 or OG2' refers to plasticizer (either sorbitol or glycerol) with 2\% MMT, the last two digitals '10' refer to the amount (wt\%) of plasticizer within the samples. For instance, OS210 stands for a 10\% sorbitol with 2\% MMT sample.

\subsection{Small angle $X$-ray scattering (SAXS)}

Small Angle X-ray Scattering was carried out at beamline BL40B2 of Spring-8 synchrotron facility in Hyogo, Japan. Detailed information of beamline BL40B2 please see Ref. [11]. The beam was monochromatized to a wavelength of $0.1 \mathrm{~nm}$ with an object distance of $1151.767 \mathrm{~mm}$. All the patterns recorded on CCD camera were calibrated by the diffraction rings from AgBH standard sample. One aluminum filter block was employed to decrease the strength of X-ray and obtain an optimized experimental pattern. The measurement time per sample was chosen to eliminate radiation damage and subsequently determined to be $10 \mathrm{~s}$. The diffraction profiles were normalized to the beam intensity and corrected considering the empty sample background. Data reduction of obtained the 2-D X-ray scattering patterns was undertaken with NIKA macros [12] based on Igor (Wavemetrics, Lake Oswego, Oregon). All recorded $Q$ vector $v s$ intensity plots were recorded after the subtraction of background.

\subsection{Differential scanning calorimetry(DSC)}

Thermal measurements using DSC were performed on 8-11 mg samples using SEIKO 6200 (Seiko, Japan) with custom analytical software. Samples were heated from $-50{ }^{\circ} \mathrm{C}$ to $250{ }^{\circ} \mathrm{C}$ at a heating rate of $5^{\circ} \mathrm{C} \mathrm{min}^{-1}$. The Avrami equation, which is widely applied in investigating crystallisation behaviors of polymer systems [13], was employed to study the crystallisation kinetics of the prepared bionanocomposites [14].

$X_{t}=\int_{t_{0}}^{t} \frac{\mathrm{d} H_{c}}{\mathrm{~d} t} \mathrm{~d} t / \int_{t_{0}}^{t_{\infty}} \frac{\mathrm{d} H_{c}}{\mathrm{~d} t} \mathrm{~d} t$

All the curves had a partial sigmoid shape, and the analysis of the development of relative crystallinity could be done using $X_{t}=1-\exp \left(-k t^{n}\right)$. A plot of $\log [-\ln (1-\mathrm{Xt})]$ vs. $\log (\mathrm{t})$ provided $n$, the value depending upon the mechanics of nucleation and on the form of crystal growth, and $k$, a rate constant containing the nucleation and growth parameters.

\subsection{Positron Annihilation Lifetime Spectroscopy (PALS)}

The PALS experiments were carried out with the National Positron Beamline Facility at the Centre for Antimatter-Matter Studies (Canberra, Australia). Positrons were implanted at $5 \mathrm{keV}$ energy to ensure that all surface effects were minimized and this energy was sufficient to implant the positrons into the bulk of the matrix. This gives a mean implantation depth of $190 \mathrm{~nm}$ into the sample, with the tail of the positron implantation profile extending up to base on density of $1.2 \mathrm{~g} \mathrm{cc}^{-1}$ (Fig. 1(a)). Each measurement consisted of $1 \times 10^{6}$ detected annihilation events and took approximately $6 \mathrm{~h}$ of measurement time. For the analysis of the PALS data, a fitting routine convoluted an instrument function 
Table 1

PALS analysis results and Avrami equation analysis results for all the prepared samples.

\begin{tabular}{|c|c|c|c|c|c|c|c|c|}
\hline \multirow[t]{2}{*}{ Sample ID } & \multirow[t]{2}{*}{$T(\mathrm{~ns})$} & \multirow[t]{2}{*}{ Weight } & \multirow[t]{2}{*}{ Pore radius $(\mathrm{nm})$} & \multirow[t]{2}{*}{ Basal spacing $\AA$} & \multirow[t]{2}{*}{$T_{\mathrm{g}}\left({ }^{\circ} \mathrm{C}\right)^{\mathrm{a}}$} & \multirow[t]{2}{*}{$X_{c} \%$} & \multicolumn{2}{|c|}{ Avrami equation parameters } \\
\hline & & & & & & & $n$ & $k$ \\
\hline OPS & $1.93 \pm 0.1$ & $12.6 \pm 1.0 \%$ & 0.28 & & 49.5 & 7.48 & 0.48 & -1.47 \\
\hline 2\% Clay & $1.92 \pm 0.1$ & $12.2 \pm 1.0 \%$ & 0.28 & 18.02 & 60.2 & 8.23 & 0.85 & -0.144 \\
\hline OG010 & $1.85 \pm 0.1$ & $15.0 \pm 1.0 \%$ & 0.27 & & 40.7 & 3.93 & 0.43 & -1.07 \\
\hline OG020 & $1.89 \pm 0.1$ & $15.7 \pm 1.0 \%$ & 0.28 & & 40.3 & 4.53 & 0.29 & -0.87 \\
\hline OG210 & $1.85 \pm 0.1$ & $10.5 \pm 1.0 \%$ & 0.27 & 19.03 & 46.7 & 9.28 & 0.20 & -0.63 \\
\hline OG220 & $1.69 \pm 0.1$ & $15.5 \pm 1.0 \%$ & 0.26 & 19.57 & 44.8 & 8.08 & 0.18 & -0.5 \\
\hline OS010 & $1.67 \pm 0.1$ & $13.7 \pm 1.0 \%$ & 0.25 & & 45.7 & 5.28 & 0.56 & -6.24 \\
\hline OS020 & $1.86 \pm 0.1$ & $10.0 \pm 1.0 \%$ & 0.27 & & 25.8 & 3.76 & 0.68 & -0.96 \\
\hline OS210 & $1.49 \pm 0.1$ & $14.9 \pm 1.0 \%$ & 0.23 & 19.80 & 47.7 & 4.01 & 0.40 & -0.78 \\
\hline OS210 & $21.2 \pm 0.1$ & $4.6 \pm 1.0 \%$ & 0.90 & & & & & \\
\hline OS220 & $1.99 \pm 0.1$ & $9.8 \pm 1.0 \%$ & 0.28 & 20.09 & 31.3 & 6.11 & 1.35 & -2.91 \\
\hline
\end{tabular}

a Data obtained from Refs. [5,10].

measurement with a series of lifetimes to determine the best parameters using in-house developed software (CAMSFIT V 4.95), an example is shown in Fig. 1(b). Analysis is performed under a variety of conditions to ensure the reliability of the results. In this case, a distribution of lifetimes is reported, reflecting the range of pore sizes within the polymer studied.

The short lifetime components have been converted to pore radii using the Tao-Eldrup model, while longer components have converted using the Rectangular Tao-Eldrup model [15]. In these measurements, the lifetime of ortho-positronium (oPs), $\tau$, can be quantitatively related to the radius of the free-volume holes $\left(r_{\mathrm{h}}\right)$. The relationship between $\tau$ and the average pore radius, $r_{\mathrm{h}}$, can be expressed by the Tao-Eldrup equation $[16,17]$ :

$\tau(n s)=\left\{2\left[1-r_{h} / \Sigma+\sin \left(2 \pi r_{h} / \Sigma\right)\right]\right\}^{-1}$

where, $\Sigma=r_{h}+\Delta ; \Delta=0.166 \mathrm{~nm}$ is an empirical parameter.

\section{Result and discussion}

The 'free-volume' is the most important fundamental parameter in a semi-crystalline polymeric system where the crystalline domains are randomly distributed throughout the polymer amorphous network, such as the starch-based polymer system. The term 'free-volume' generally refers to the total unoccupied volume, made up of various 'holes' with different shapes and sizes, and are caused by the irregular molecular packing, density fluctuations and topological constraints. As mentioned in the introduction, such 'holes' can be observed as the different lifetimes of positrons in a PALS measurement; and the lifetime distribution generated from the PALS could further elucidate the distribution of such 'holes', with varying size and weight, throughout the polymeric network.

We have recently found that the molecular interactions existed within the starch-MMT system are complex because they influence the re-organization and re-configuration of the polymer crystalline domains based on their concentration and the component-ratio [5]. The glass transition temperature and the bulk crystallinity of starch/plasticizer/MMT ternary systems for two plasticizers; glycerol and sorbitol are reported in our previous reports [10,18]. And since there is little evidence of direct correlation between these two fundamental properties, we further analyzed the crystallization kinetics to understand the growth and assemblage of the crystalline domains using Avrami analysis in terms of two parameters (' $n$ ' and ' $k$ ') because these parameters convey information about the nucleation mechanism and growth of crystalline domains $[5,10]$, as shown in Table 1 . In this paper, we will attempt to relate the void sizes and void distribution to the characteristics of the crystalline domains for both prepared samples.

Based on the free-volume plasticization theory, the plasticizer molecules in the matrix would modify the organization of the polymeric chains and thus influence the formation of crystalline domains [19]. In other words, any reconfiguration of the crystallinity within the polymeric network can be reflected as changes to the system free-volume, and the distribution of free-volume. The PALS technique has proven relevance in polymer science with an aim to understand the free-volume related phenomenon. More detailed discussion on how the free-volume changed across different plasticizer (type and amount) and MMT will be presented in the following section.

\subsection{Study of the binary polymeric system}

\subsubsection{Starch + nanoclay system ( $2 \%$ clay)}

For various reasons, including cost and processing, in industrial applications utilizing nanoclays or nanoparticles, $2 \mathrm{wt} \%$ is considered to be quite typical as long as the particles have successfully intercalated within the polymeric system. Therefore, it was necessary to investigate bionanocomposites systems have sufficient intercalation of the MMT. Because the processing route for dispersion of MMT in polymers is a mature field, all samples showed excellent intercalation and/or exfoliation of MMT $[5,10]$. The PALS analysis showed that the addition of MMT did not significantly changed the size of the free-volume pore within the polymeric system, as measured through the changes to the lifetime analysis of the positron annihilation (data shown in Table 1). It presents an interesting proposition for the free-volume through the 'hole-filling' mechanism, proposed in Townrow et al.'s work, where the water molecules filled the void spaces amongst the polymer chains [20]. In the present study, the interactions occupied certain amount of the open volume that detectable in the starch network, representatively with a pore size of $0.28 \mathrm{~nm}$, Table 1 . Such results strengthen the argument that the molecular level mixing of the MMT and the starch polymer chains had re-organized the polymeric packing and redistributed the free-volume. In terms of the bulk properties, a change in the distribution of the matrix freevolume reflects on the bulk crystallinity. Thus, we observe that crystallinity of the $2 \%$ wt MMT sample (Table 1 ) increased from $7.48 \%$ (OPS) to $8.23 \%$ (2\%Clay) as the MMT could provide greater nucleation sites to form crystalline domains.

Furthermore, when we compare this result with the glass transition result presented earlier, we can also see evidence of strong reinforcing effect due to the MMT through the increased $T_{\mathrm{g}}$ $\left(60.2^{\circ} \mathrm{C}\right.$ ) when compared to the pure polymer matrix (OPS sample with a $T_{\mathrm{g}}$ of $49.5^{\circ} \mathrm{C}$ ), seen in Table 1. 

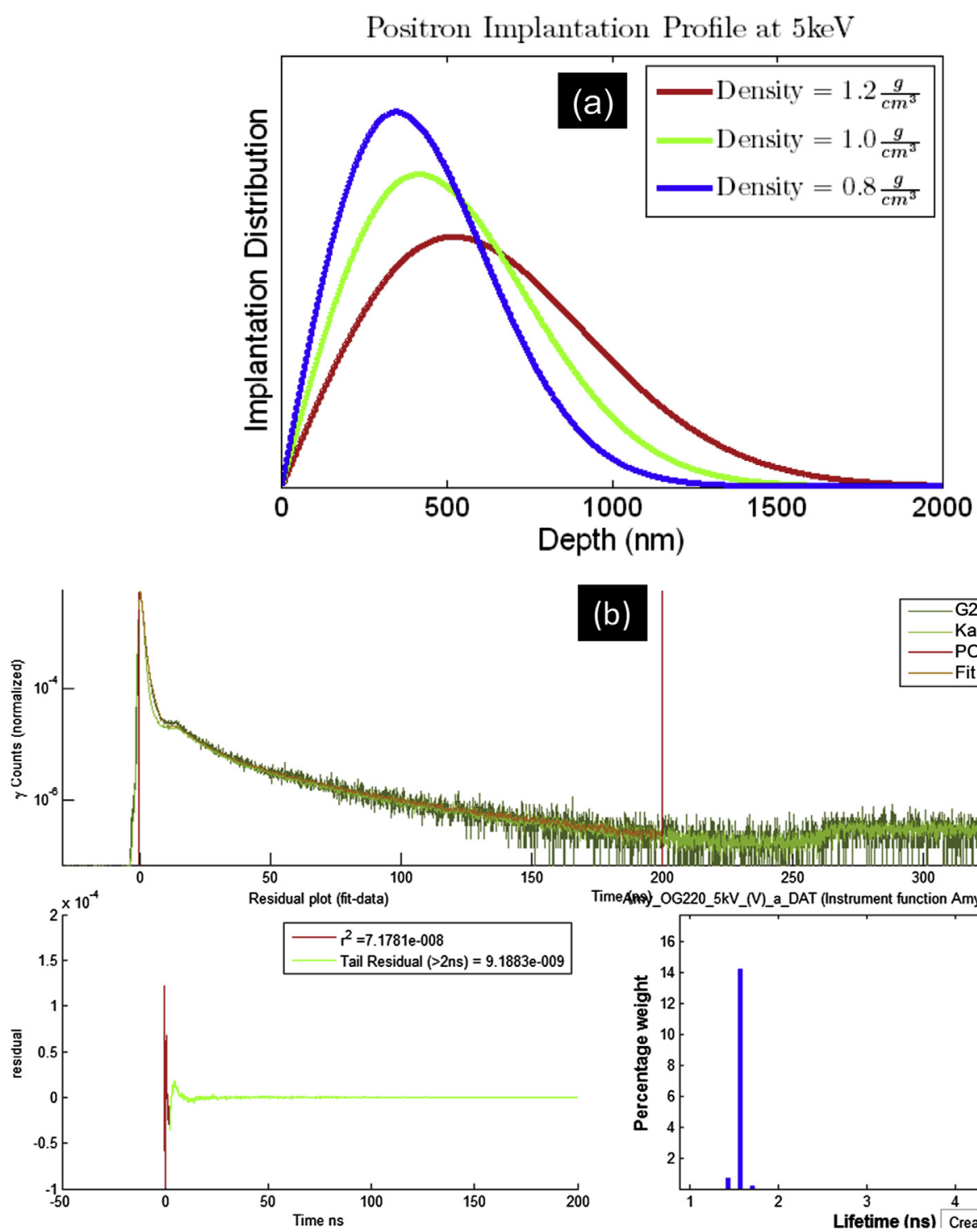

(b) TimeAnol_OG220_5kV_(V)_a_DAT (Instrument function Amy_kapton_5kV_(X)_a_HDR)

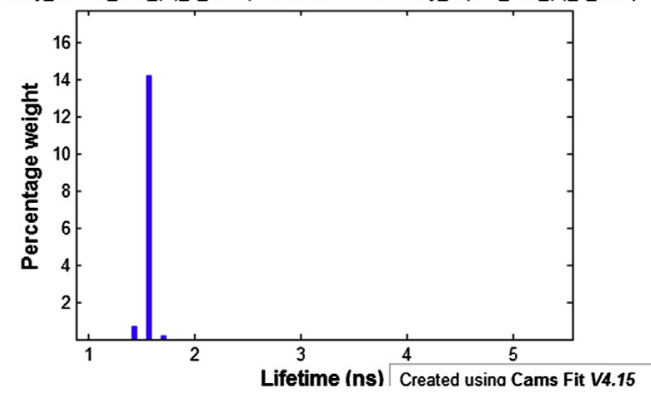

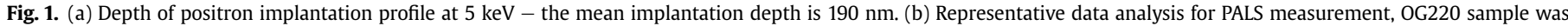
presented here.

\subsubsection{Starch and plasticizer system}

As expected, Table 1 shows that the addition of either plasticizer (glycerol or sorbitol) modified the free-volume within the polymer matrix and further, it is also evident that the extent of free-volume modification is related to the plasticizer concentration. As the plasticizer molecules occupy the void space between polymer chains, they influence the organization of the polymeric chains and alter the pore volume distribution. Thus, one interesting aspect is the slight increase in the actual pore-size upon increasing plasticizer content in the starch + plascizier samples, which is an indication of greater molecular packing where when the molecular are greater compacted, the space between molecules tended to increase. Also, evidence of the molecular interactions within the plasticized matrix can be seen through a comparison of the total free-volume weight of plasticized samples. The total free-volume was larger than that of the pure starch sample, except the OS020 sample. The increase is a well-known phenomenon, called the typical plasticization effect, widely accepted to be related to the free-volume theory. However, it should be note that, the plasticizing effect of glycerol and sorbitol is obvious different to each other. The increase of glycerol did not cause either big change in the pore radii or relative weight of the open volume, as read from Table 1 . The pore radii and relative weight are similar to OG010 and OG020. Differently, comparing OS010 and OS020, an increase in pore radii (from $0.25 \mathrm{~nm}$ to $0.27 \mathrm{~nm}$ ) and decrease in relative weight are observed. The reduction in free-volume with further increase in the sorbitol concentration is an interesting finding. In the case of the $20 \%$ sorbitol sample, total free-volume decreased from $13.7 \%(\mathrm{OS} 010)$ to $10.0 \%(\mathrm{OSO} 20)$ in Table 1 . We might consider the possibility that if sorbitol/sorbitol interactions become stronger and compete with the starch/sorbitol interactions, they would weaken the extent of plasticization from sorbitol, and thus reduce the overall free-volume of the matrix. Therefore, through these significant changes in the pore sizes, these results demonstrate the strong plasticizer/plasticizer interactions which hindered the plasticizer/starch interactions. Such assumption had been previously mentioned in our reported $[5,10]$.

\subsection{Ternary polymeric system (starch/plasticizer/nanoclay)}

A ternary system, involving a polymer matrix and few additives, represents the 'real-world' packaging film and in such a system, the 
resolution of different interactions is complicated by the presence of interactions between the plasticizers and the nanoclays. As an example, the $2 \%$ wt MMT sample with no plasticizer had an overall relative weight of $12.2 \%$, but the addition of either plasticizer (glycerol or sorbitol) increased the total free-volume of the sample. As read from Table 1 the total free-volume increased from $12.2 \%(2 \%$ Clay sample) to $13.7 \%$ (OS210) and $15.0 \%($ OG210), respectively. Together with the presence of plasticizer, the addition of nanoparticle, MMT, significantly altered the free-volume distribution in the corresponding samples. The ternary interactions in the polymeric system are not only dependent on either individual component but also on the interplay among the components via considering the possible interaction. For example, in the starch/ glycerol/MMT system, the pore radii decrease upon the increasing of glycerol amount, but the relative weight did increase with the increasing glycerol concentration, Table 1 . This observation suggested that the addition of MMT facilitated the plasticization effect of glycerol which resulted in a decreased pore radius. This is the decreased pore radii could be resulted from a more flexible polymeric network that accommodates more glycerol molecules between the polymer chains. On the other hand, the presence of the MMT increased the quantity of the glycerol could be accommodated due to its one dimensional barrier effect in starch network. Meanwhile the rearrange (de-tangle) process that the starch
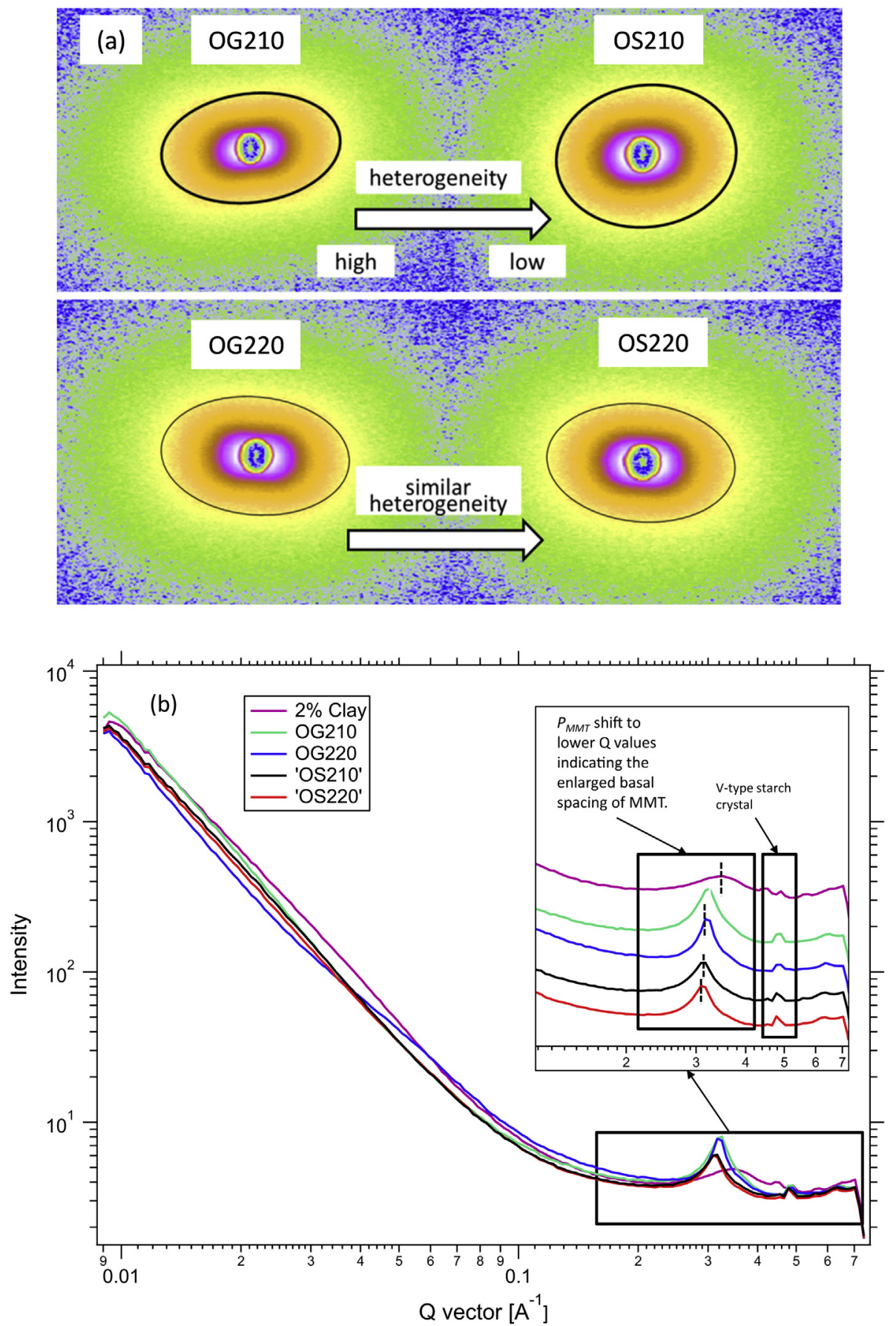

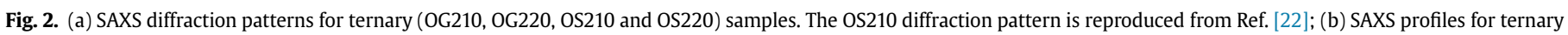
(OG210, OG220, OS210 and OS220) samples. 
network become more flexible is accompany with freeing some inherit open-volume from the starch chain itself. Thus the total relative weight of the open volume is found to increase upon the increasing of glycerol concentration. The hypothesis (addition of MMT, increased the relative weight but decreased the pore radius) is more pronounce in the sorbitol plasticizer case. The addition MMT(2\%) in the OS010 system, promote the emergence of a new dateable pore radius $(0.90 \mathrm{~nm})$, and the total amount significantly increased from 13.70 (OS010) to $19.5 \%(\mathrm{OS} 210)$ but the pore radius are smaller. On the other hand, in the crystallinity result analysis, unique finding that the addition of MMT did not as expected but decrease from $5.28 \%$ (OS010) to $4.01 \%$ (OS210) after the addition of $2 \%$ MMT. This could possibly relate to the unique finding in the free volume distribution for OS210 where two pore radius was detected in OS210 $(0.23 \mathrm{~nm}$ and $0.90 \mathrm{~nm})$. However, this assumption needs to be studied by further experiments. However, different finding comes from the OS220 sample, where the relative weight and pore radius both decreased with increasing the sorbitol concentration from $10 \%$ to $20 \%$, Table 1 . The different finding in terms of the pore size and distribution in the OS210 and OS220 strongly suggested that the crystalline domain size and molecular packing of the starch/MMT/sorbitol assembles this is reflected as the crystallinity shown in Table 1.

Specifically, the impact of excess plasticizer on pore radius changes becomes more pronounce in the presence of MMT which implying the complex interaction between plasticizer and MMT. By comparing the data from different plasticizer samples, some insights of the effect of MMT on polymer rearrangement could be noticed. It is seen that at higher plasticizer concentration, the pore radius are overall larger than that from the low plasticizer samples regardless the plasticizer type. This is the impact of two key phenomena; the 'hole-filing' process, and the increased interactions between plasticizer/plasticizer, which was discussed in detail in the previous sections. The key conclusion from this analysis is that with lower plasticizer loading, the nanoclay would dominate due to its nucleating effect and increase the free-volume; however a balance could be found by varying the plasticizer amount. This is supported by higher crystallinity and larger crystal size found in lowplasticizer samples (Table 1). It has been demonstrated in previous studies that the plasticizer-size influences the interactions of plasticizer/nanoclay and plasticizer/starch, and hence influences the formation of various crystalline domains [10,21].

Also, when the glass transition and the thermal analysis is taken into account, Table 1 data showed slightly higher ' $n$ ' values in Avrami analysis for sorbitol samples, indicating strong onedimensional crystalline growth and heterogeneity. Since larger molecular size of a plasticizer usually favored the growth of single crystalline domains [5], we find the effect of larger crystalline domains through this PALS study. For example, sorbitol-plasticized sample showed a relatively smaller pore-size with higher relative weight (Table 1) imaging the fact that smaller neighboring open volume would be when stronger heterogeneity existed in the polymeric network which could be mutually reflected as the new emerge large pore radius $(0.90 \mathrm{~nm})$ in OS210. The heterogeneity in the crystalline domain can be seen through the SAXS analysis, shown in Fig. 2. As shown in Fig. 2, when the plasticizer concentration is $10 \mathrm{wt} \%$, glycerol and sorbitol showed varying degree of heterogeneity - depending upon a balance of interactions - but as the concentration increases to $20 \mathrm{wt} \%$, the similarity in the ellipticity indicated similar domain heterogeneity for both OG220 and OS220.

The Bragg peaks occurred at a series of $Q$ values satisfying the relationship $d \approx 2 \pi / Q(\mathrm{~nm})$. The corresponding basal spacing values are shown in Table 1 together with the characteristic parameters for each sample. As read from Table 1, sorbitol plasticized samples showed an overall higher basal spacing ( $d$-spacing) than glycerol samples. And the basal spacing value increased upon the increase of plasticizer concentration. The basal spacing for OG210, OG220, OS210 and OS220 is $19.04 \AA, 19.57 \AA, 19.8 \AA$ and $20.03 \AA$, respectively. This could be largely attributed to the relative larger size of sorbitol when comparing to glycerol molecules. This assumption had been discussed in detailed in our previous publications $[5,10,18]$.

Furthermore, the variation in pore radius and relative weight can be supported from the $T_{\mathrm{g}}$ results and thermal analysis results, as shown in Table 1. For example, the relative high $T_{\mathrm{g}}$ obtained in the DSC measurements for low sorbitol concentration samples indicated closer packing of the crystalline domains, which would be translated to the reduction in pore radius observed and increase in relative weight (heterogeneity) as compared to the glycerolplasticized samples. Moreover, the larger $n$ values from the Avrami analysis for sorbitol-plasticized samples indicated stronger heterogeneity as well. Since it is common knowledge that higher heterogeneity would give a closer packing, either with the polymer/polymer interaction or with the polymer/plasticizer interaction, they corroborate the relatively smaller pore radius in the corresponding samples, Table 1 . However, different tendency had been observed when the sorbitol concentration increased to $20 \%$.

\section{Concluding remarks}

Positron Annihilation Lifetime Spectroscopy (PALS) experiments using Beamline 9940 at Centre for antimatter-matter studies (Canberra, Australia) were carried out to study the MMT and plasticizer (type and concentration) interactions with starch polymer at the molecular-scale.

It is demonstrated that the complexity within the molecular-scale interactions in the ternary bionanocomposite system arise due to the interplay between the MMT and the plasticizer (type and concentration). It's found that addition of MMT did not alter either the free volume radii or the relative weight \% of the positrons that annihilate within those free volume pores with the absence of plasticizer. In the starch-plasticizer system, one interesting aspect is the increase in the actual pore-size upon increasing plasticizer content. This is an indication of greater molecular packing where when the molecular are greater compacted, the space between molecules tended to increase.

Moreover, the samples with both MMT and plasticizer clearly demonstrate ternary interactions as seen in OG210 or OG220 as well as in OS210 and OS220. For example, in the starch/glycerol/ MMT system, the pore radii decrease upon the increasing of glycerol amount, but the relative weight did increase with the increasing glycerol concentration. The hypothesis (addition of MMT, increased the relative weight but decreased the pore radius) is more pronounce in the sorbitol plasticizer case. The addition MMT in the OS010 system, promote the emergence of a new dateable pore radius $(0.90 \mathrm{~nm})$, and the total amount significantly increased from 13.70 (OS010) to 19.5\% (OS210) but the pore radius are relatively smaller $0.23 \mathrm{~nm}$ compared to OS010 $(0.25 \mathrm{~nm})$. Finally, the results are mutually supported from the thermal analysis and SAXS data and crystal growth analysis using Avrami analysis.

\section{Acknowledgments}

The SAXS experiments were carried out at Spring-8 with help from Dr. Kyuya Nakagawa (University of Hyogo). Dr. HuiHua LIU thanks the AINSE award and acknowledges the internal funding from Curtin University. Dr. Liu also acknowledges the internal funding and the Collaborative Research Network (CRN) from 
Federation University Australia. There is no potential conflict of interest between the researchers and this investigation does not bias any other investigation.

\section{References}

[1] D.S. Chaudhary, Competitive plasticization in ternary plasticized starch biopolymer system, J. Appl. Polym. Sci. 118 (2009) 486-495.

[2] D. Chaudhary, B. Adhikari, S. Kasapis, Glass transition behaviour of plasticized starch biopolymer system-a modified Gordon-Taylor approach, Food Hydrocoll. 25 (2010) 114-121.

[3] D.S. Chaudhary, B.P. Adhikari, Understanding polymeric amylose retrogradation in presence of additives, J. Appl. Polym. Sci. 115 (2010) 2703-2709.

[4] F. Chivrac, E. Pollet, P. Dole, L. Avérous, Starch-based nano-biocomposites: plasticizer impact on the montmorillonite exfoliation process, Carbohydr. Polym. 79 (2010) 941-947.

[5] H. Liu, D. Chaudhary, J. John, M.O. Tade, Morphological investigation into starch bionanocomposites via synchrotron radiation and differential scanning calorimetry, J. Nanotechnol. 2011 (2011).

[6] A. Hill, M. Zipper, M. Tant, G. Stack, T. Jordan, A. Shultz, A free volume approach to the mechanical behaviour of miscible polycarbonate blends J. Phys. Condens. Matter 8 (1996) 3811.

[7] M. Zipper, G. Simon, P. Cherry, A. Hill, The effect of crystallinity on chain mobility and free volume in the amorphous regions of a miscible polycarbonate/polyester blend, J. Polym. Sci. Part B: Polym. Phys. 32 (1994) 1237-1247.

[8] Y.C. Jean, P.E. Mallon, D.M. Schrader, Principles and Applications of Positron \& Positronium Chemistry, World Sci. Pub Co. Inc. (2003) 329-348.

[9] M. Roussenova, M. Murith, A. Alam, J. Ubbink, Plasticization, antiplasticization, and molecular packing in amorphous carbohydrate-glycerol matrices, Biomacromolecules 11 (2010) 3237-3247.

[10] H. Liu, D. Chaudhary, S. Yusa, M.O. Tadé, Glycerol/starch/Na+-montmorillonite nanocomposites: a XRD; FTIR; DSC and 1H NMR study, Carbohydr. Polym. 83 (2011) 1591-1597.
[11] K. Inoue, T. Oka, K. Miura, N. Yagi, Present status of BL40B2 and BL40XU at SPring-8 (Beamlines for small angle X-ray scattering), in: AIP Conference Proceedings, San Francisco, California, 2004, p. 336.

[12] C.S. Kealley, M.M. Elcombe, R. Wuhrer, E.P. Gilbert, Application of small-angle scattering to study the effects of moisture content on a native soy protein, J. Appl. Crystallogr. 41 (2008) 628-633.

[13] S.W. Lee, M. Ree, C.E. Park, Y.K. Jung, C.S. Park, Y.S. Jin, D.C. Bae, Synthesis and non-isothermal crystallization behaviors of poly(ethylene isophthalate-coterephthalate)s, Polymer 40 (1999) 7137-7146.

[14] D.S. Chaudhary, R. Prasad, R.K. Gupta, S.N. Bhattacharya, Clay intercalation and influence on crystallinity of EVA-based clay nanocomposites, Thermochim. Acta 433 (2005) 187-195.

[15] J.P. Sullivan, J. Roberts, R.W. Weed, M.R. Went, D.S. Newman, S.J. Buckman, A trap-based positron beamline for the study of materials, Meas. Sci. Technol. 21 (2010) 085702.

[16] S.K. Sharma, G. Roudaut, I. Fabing, G. Duplâtre, Characterization of a sucrose starch matrix through positron annihilation lifetime spectroscopy: unravelling the decomposition and glass transition processes, Phys. Chem. Chem. Phys. 12 (2010) 14278-14284.

[17] M. Eldrup, D. Lightbody, J. Sherwood, The temperature dependence of positron lifetimes in solid pivalic acid, Chem. Phys. 63 (1981) 51-58.

[18] H. Liu, D. Chaudhary, S. Yusa, M.O. Tade, Preparation and characterization of sorbitol modified nanoclay with high amylose bionanocomposites, Carbohydr. Polym. 85 (2011) 97-104.

[19] A.K. Doolittle, Mechanism of Plasticization, Plasticizer Technology, Reinhold, New York, 1965

[20] S. Townrow, D. Kilburn, A. Alam, J. Ubbink, Molecular packing in amorphous carbohydrate matrixes, J. Phys. Chem. B 111 (2007) 12643-12648.

[21] H. Liu, D.S. Chaudhary, M.O. Tadé, Effect of Nanoclay and Sorbitol on Structure Relaxation of Extruded High-amylose Bionanocomposite via Synchrotron Radiation, CHEMECA, Sydney, Australia, 2011, pp. 1675-1684.

[22] H. Liu, D. Chaudhary, J. Roberts, R. Weed, J. Sullivan, S. Buckman, The interaction in sorbitol-plasticized starch bionanocomposites via positron annihilation lifetime spectroscopy and small angle X-ray scattering, Carbohydr. Polym. 88 (2012) 1172-1176. 